\title{
Assessing hyolaryngeal excursion: Comparing quantitative methods to palpation at the bedside and visualization during videofluoroscopy
}

\author{
Danielle Brates, $\mathbf{M S}^{1,2}$, Sonja M Molfenter, $\mathbf{P h D}^{1}$, and Susan L. Thibeault, $\mathbf{P h D}^{2}$ \\ ${ }^{1}$ Department of Communicative Sciences and Disorders, NYU Steinhardt, 665 Broadway, 9th \\ Floor, New York, NY 10012 \\ ${ }^{2}$ Division of Otolaryngology-Head and Neck Surgery, University of Wisconsin, School of Medicine \\ and Public Health, Wisconsin Institutes for Medical Research (WIMR) BLDG. 1485, 1111 \\ Highland Avenue, Madison, WI 53705-2275
}

\section{Introduction}

During a normal swallow, contraction of the suprahyoid muscles results in anterior and superior movement of the hyoid bone and larynx [1]. This movement displaces the larynx from the bolus pathway and contributes to bolus clearance through the upper esophageal sphincter [2,3]. It is believed that inadequate hyolaryngeal excursion (HE) may result in incomplete airway closure and subsequent penetration, aspiration [4], and/or reduced opening of the upper esophageal sphincter resulting in pharyngeal stasis [5]. Although videofluoroscopy is required for direct visualization of these processes, speech-language pathologists often initially rely on clinical information to assess an individual's degree of swallowing impairment, aspiration risk, and the need for further testing.

A clinical swallowing exam (CSE) typically serves as the initial assessment of swallowing ability [6,7]. A standard CSE protocol includes a case history, an examination of cranial nerve function, and swallow testing with food and liquid, if appropriate. While administering solids and liquids of varying consistencies and volumes, clinicians palpate the hyolaryngeal complex. Logemann developed and popularized the four-finger method of palpation, wherein the first finger is placed on the submental muscles, the second finger on the hyoid bone, and the third and fourth fingers on the thyroid cartilage as the patient is instructed to swallow [8]. Per Logemann, the purpose of this positioning is to assess the strength of the tongue, hyoid, and laryngeal movements during swallowing. Additionally, these structures are palpated with the intention of making a gross estimation of oral transit time and

\footnotetext{
Corresponding author: Susan L. Thibeault, PhD, thibeault@surgery.wisc.edu, Phone: (608) 263-0121, Fax: (608) 263 -6751. db2814@nyu.edu, Phone: (914)621-1819, smm16@nyu.edu, Phone: (212)992-7694

Compliance with Ethical Standards:

Conflict of Interest: The research conducted by the first author was supported by funding from the Diane M. Bless Endowed Chair in Otolaryngology.

Ethical approval: All procedures performed in studies involving human participants were in accordance with the ethical standards of the Institutional Review Board and with the 1964 Helsinki declaration and its later amendments or comparable ethical standards. Informed consent: Informed consent was waived with IRB approval for retrospective medical chart review data collection.
} 
pharyngeal delay based on the relative timing of structural movements [8]. Reduced HE to palpation is clinically adopted as a sign of impaired laryngeal elevation and closure, and a potential indication of reduced swallow safety [9].

All aspects of swallow function assessed during the CSE require a certain degree of subjective interpretation. In particular, evaluation of HE may be at heightened risk for clinician bias. Several factors have been shown to affect HE, both within-subject (bolus volume [10-13], consistency [9], and taste [14,15]), and between-subject (sex [16] and age [17]). Furthermore, Molfenter and Steele demonstrated a high degree of variability in reported norms for HE even when controlling for the above factors [18]. One factor contributing to discrepancies in normative data may be measurement error due to known variability in selection of a hyoid rest frame, which is needed for calculating rest-to-peak hyoid displacement. Peak values (most commonly peak anterior, peak superior, and peak hypotenuse positions) have been demonstrated to be sufficient for capturing hyoid movement, and eliminate the need for rest frame selection [18].

Another potential source of variation specific to the tactile nature of palpation may be the physical feasibility of this technique. Increased submental adipose tissue can impede adequate appreciation of laryngeal movement, or the presence of a cervical collar may limit access to the hyolaryngeal complex.

Given the potential for significant within- and between-subject variation in HE as well as external barriers to palpation, it would seem a significant challenge for clinicians to assess adequacy of HE based on palpation alone. Nevertheless, McCullough et al. found that $62.1 \%$ of speech-language pathologists report using the four-finger palpation method during swallowing evaluations in a neurogenic population [19]. The validated and widely used Mann Assessment of Swallowing Ability (MASA) relies on palpation to judge the highest point of hyolaryngeal movement as either complete, mildly restricted, incomplete, or absent [20].

Despite the widespread use of palpation among practicing clinicians, minimal evidence exists to support its utility. Across three consistencies (thin liquid, thick liquid, and puree), McCullough et al. found insufficient inter-rater reliability for determining decreased hyolaryngeal elevation via palpation; intra-rater reliability was sufficient only for thin liquid [21]. Rangarantham and McCullough reported that perceptual rating of laryngeal elevation at the bedside (technique unspecified) was significantly associated with perceptual rating of hyolaryngeal excursion during VFSS based on a 4-point perceptual rating scale [22]. However, Lee and colleagues demonstrated that compared to normative data, experienced raters correctly distinguish between "normal" and "abnormal" hyoid elevation on videofluoroscopic swallow studies (VFSS) only $61.3 \%$ of the time, with low inter-rater agreement $(\mathrm{k}=0.40)$ [23].

The aim of this study was to compare clinician ratings of HE to quantitative measures of hyoid movement to determine whether significant differences in hyoid movement are present when HE is perceptually judged to be reduced versus normal. Specifically, we compared quantitative biomechanical movement of the hyoid on VFSS with two groups: binary 
judgment of hyoid movement adequacy at the bedside (palpation) and binary judgement of hyoid movement adequacy in the VFSS (visualization). We hypothesized no significant difference in the quantitative hyoid measures between swallows judged to be reduced compared to those judged to be normal in either perceptual category (palpation, visualization). The analysis was structured in these terms to avoid the need for comparison to normative HE data, which, as previously mentioned, is problematic due to high variability. Three methods of hyoid measurement were used: peak anterior position, peak superior position, and the combined peak hypotenuse hyoid position. An additional exploratory aim was to investigate the influence of rater experience on clinician judgment of HE.

\section{Methods}

\section{Participants}

This study was approved by the University of Wisconsin Madison School of Medicine and Public Health Institutional Review Board. A medical chart review was conducted of inpatients referred for a swallow evaluation at the University of Wisconsin Hospital and Clinics. Beginning in November 2016, charts meeting inclusion criteria were reviewed in reverse chronological order until a convenience sample of 100 cases was reached. This target sample size was based on a power calculation indicating a minimum sufficient sample size of 76 (see below) and anticipatory exclusion of studies based on image quality and missing data. Adult inpatient records were reviewed and considered for inclusion if a CSE and VFSS were both conducted as part of standard of care within a 24- hour period. Subjects were excluded if a puree bolus was not administered during the VFSS. Demographic data extracted from the medical record included sex, age at time of evaluation, hospital service, height, and BMI. Of the 100 total patient records collected,13 were excluded based on poor landmark visibility on VFSS (e.g. cervical hardware obstructing cervical spine landmarks) or piecemeal swallow of the bolus of interest. This left 87 patient records that were included for analysis (56 males; mean age $61.42 \pm 19.03$ ).

\section{CSE and VFSS Ratings}

Clinicians' ratings of HE were collected from CSE and VFSS reports at the time of evaluation. On the CSE flowsheet that populates all standard-of-care evaluation reports at this clinical site, a list of potential impairments includes "reduced hyolaryngeal rise," which could be checked or left unchecked by the clinician. Thus, if observations of impairment were omitted from the clinician report, is was assumed that the clinician had judged HE to be normal.. On VFSS reports, a forced choice binary rating of HE as either "reduced" or "within functional limits" was documented for all patients. For the purposes of this study, "within functional limits" was operationalized to be normal HE based on clinical judgment. Because of the retrospective design, it was not known whether clinicians made judgments in real time or chose to review VFSS studies prior to their final interpretation. However, all studies were recorded at 30 frames per second on a picture archiving and communication system (PACS) and redundantly on DVD so all clinicians had the option of frame by frame review in the original frame rate. For both the CSE and VFSS, a single, global judgment of HE was made for each participant based on an overall impression of HE across all swallow 
trials. Subjects were grouped based on the binary rating in both assessment settings, so that each participant had an assignment of either R (reduced) or N (normal) for both the CSE and VFSS assessment. These participant groups are henceforth defined as R-CSE (reduced on palpation) and N-CSE (normal on palpation) and R-VFSS (reduced on visual inspection during VFSS) and N- VFSS (normal on visual inspection during VFSS).

The clinician performing the evaluations varied across participants. In most cases, the same clinician performed both exams, though instances in which two different clinicians conducted the assessments were also included in the sample. All clinicians were either certified speech language pathologists or speech language pathology clinical fellows (i.e. up to one year of clinical experience). Clinician raters were separated into three categories based on number of years experience, shown in Table 1. These categorical groupings were made for two reasons. First, separating clinical fellows who were not yet licensed SLPs and were still in training appeared to be a logical demarcation. Next, the 10 year cut-off point was made in an effort to evenly distribute the number of SLP raters in each category.

\section{HE Measurement Collection}

All VFSSs were recorded at 30 frames per second. The initial puree bolus (Varibar® Pudding barium sulfate paste) in lateral view was selected for hyoid measurement. Due to the retrospective nature of this investigation, this bolus was chosen to ensure comparable bolus volume between subjects $(\sim 5 \mathrm{ml})$ due to consistent administration of this consistency via teaspoon. Puree was also the most reliably administered consistency across swallow studies, given the varying degrees of dysphagia severity represented in this sample. Single puree swallows from each participant were clipped from the larger VFSS and de-identified for blinded, randomized measurement.

ImageJ open source software [24] was used to measure HE of all participants. To account for inherent between-subject variation, quantitative data were collected using an internal anatomical scalar as described by Molfenter \& Steele (2014), such that all hyoid measurements represented a percentage of the $\mathrm{C} 2-\mathrm{C} 4$ length to control for variation in participant height (Fig 1). With this anatomical reference as the vertical axis, peak hyoid position was measured for anterior, superior, and hypotenuse hyoid positions [25].

\section{Statistical Analyses}

A power calculation using a primary outcome of hyoid peak position determined a minimum sample size of 76. Effect size was hypothesized based on the group means and standard deviations observed in previous studies that quantified hyolaryngeal movement [26,27]. Sufficient statistical power was considered as a $90 \%$ probability to detect the statistical significance in the group difference in sample testing given that there is a true difference in the population. Intraclass correlations were used to evaluate the intra- and inter-rater reliability of all hyoid measures. Cohen's simple kappa agreement coefficient was adopted to measure the consistency between tactile perception of HE based on palpation and visual perception of HE based on VFSS. Independent-sample t-tests were applied to assess differences in peak anterior, superior, and hypotenuse hyoid positions between patients judged to have complete versus reduced hyolaryngeal rise to palpation. This analysis was 
repeated for HE ratings made during VFSS. Bonferroni correction was applied to account for multiple comparisons, resulting in an alpha level of 0.008 .

The exploratory analysis examining the effect of experience on HE rating was restricted to conditions and hyoid positions that yielded significant results in the primary analysis. Given that the number of raters and evaluations performed in each experience group were unequal, this question was tested using mixed effects logistic regression with rater as a random effect.

Additionally, a chi square test was used to determine whether agreement between binary perceptual ratings on the CSE and VFSS was related to consistency of clinician between the two exams.

\section{Results}

Participants

Eighty-seven patient reports were included in the final analysis (56 males, mean age $=61.4$, $\mathrm{SD}=19.0$ ). For males, the mean height was $177.1 \mathrm{~cm}, \mathrm{SD}=9.2$ and mean BMI was 28.2 , $\mathrm{SD}=5$.6. For females, mean height was $161.5 \mathrm{~cm}, \mathrm{SD}=8.6$, and mean $\mathrm{BMI}$ was $29.2, \mathrm{SD}=$ 8.1. The sample comprised a wide range of patient diagnoses and reasons for admission. Table 2 lists the inpatient service categories represented in the sample.

\section{Reliability}

Intra-class correlation coefficient results for intra- and inter-rater reliability on all three hyoid measurements is reported in Table 3. Across all measures, raters achieved good-toexcellent reliability (ICC>0.7) [28].

Agreement between CSE and VFSS perceptual ratings-Table 4 shows the number of participants in each clinician-rated group. Out of the 87 patients, 29 were judged to have normal HE during both the CSE and VFSS and 25 were judged to have reduced HE during both assessments (i.e., agreement in rating between CSE and VFSS). In 16 cases, HE was thought to be normal upon bedside palpation, but reduced on VFSS visualization, and 17 patients were reported to have reduced HE on palpation but normal during VFSS (both indicating disagreement between ratings). This resulted in a kappa coefficient of $\mathrm{k}=.62$, indicating moderate agreement between CSE and VFSS perceptual rating of HE.

\section{Perceptual ratings from CSE compared to objective measurements-}

Descriptive statistics for each clinician-rated group can be found in Table 5. Independent sample t-tests revealed a significant difference in anterior position between the R-CSE and $\mathrm{N}-\mathrm{CSE}$ groups $[\mathrm{t}(85)=3.30, \mathrm{p}=0.001]$ The effect size was moderate $(\mathrm{r}=0.34)$. No significant differences were observed between groups in the superior or hypotenuse position (Fig 2).

\section{Perceptual ratings from VFSS compared to objective measurements-}

Independent sample t-tests revealed no significant differences in objective hyoid position between the R-VFSS and N-VFSS groups across any hyoid position (anterior, superior, or hypotenuse) (Fig 3). There was no difference in objective peak hyoid position when 
clinicians rated swallows to be reduced compared to when they rated them to be normal based on VFSS studies.

\section{Rater Experience}

The analysis of the effect of clinician experience on HE rating was restricted to the only significant group difference observed: peak anterior position during palpation. A mixed effects logistic regression with rater as a random effect revealed that the relationship between objective hyoid measures and clinician ratings did not vary as a function of experience group (see Table 1). In terms of odds ratios, compared to clinicians with the most experience (Group 3), those with the least amount of experience (Group 1) were 2.90 times more likely to judge any given swallow as reduced based on palpation $(\mathrm{OR}=2.90,95 \% \mathrm{CI}$ : $1.02-8.22, p=.05)$.

\section{Clinician Rater for CSE and VFSS}

As detailed in Table 6, the proportion of paired CSEs and VFSSs in agreement rated by the same rater $(42 / 54=77.8 \%)$ did not differ significantly from those rated by different raters $(23 / 33=69.7 \%)$ [Pearson's $\chi 2=0.708, p=0.4]$. This finding indicates that clinicians were no more biased in their ratings of HE during the VFSS if they had performed the preceding CSE themselves.

\section{Discussion}

Perceptual judgment is typically used in clinical settings to assess adequacy of hyolaryngeal excursion (HE), though the validity of perceptual techniques for assessing this physiologic component of swallowing has not been established. In this study, binary ratings of HE, made via palpation and videofluoroscopic visualization, were compared to objective, sizecontrolled measures of hyoid movement.

As discussed earlier, there is a lack of agreement in the current literature for what constitutes normal versus impaired hyoid and laryngeal movement. Much of the discrepancy in reported norms may be attributed to the high degree of HE variability known to exist in safe, functional swallowing. Furthermore, our ability to compare to normative values is limited by the lack of a comparable reference in the literature. The vast majority of reported HE norms are based on swallows of liquid consistencies. Therefore, it would have been challenging to choose a value or even a range of values to serve as a benchmark of normal HE for quantitative comparison. This speaks to the complexity of swallowing physiology and serves as a rationale for the design used in this study. We categorized swallows based on a binary perceptual rating of reduced or normal (either via palpation or VFSS visualization), and then compared the swallows in each group based on measured, size-controlled hyoid biomechanics at the height of the swallow. Thus, this was an exploration of how and if clinicians make meaningful distinctions between the hyolaryngeal movements of a heterogeneous population using standard-of-care perceptual methods, irrespective to any external reference of "normal".

Comparing CSE to objective measurements, we found that peak anterior hyoid position was significantly lower in patients judged to have reduced HE compared to those judged to have 
functional HE. There was no significant difference in measures of peak superior or hypotenuse hyoid positions between patients judged to have reduced versus normal HE. These results suggest that palpation by the clinicians in our sample was sensitive only to differences in anterior hyoid movement. No significant differences were observed between the N-VFSS and R-VFSS groups, indicating that clinicians were not able to visually judge differences in any plane of hyolaryngeal movement (anterior, superior, or hypotenuse) during VFSS. These results are consistent with prior research by Lee and colleagues [23], and, taken together with the above findings, suggest that tactile perception may be more salient than visual for assessing HE. This is a surprising finding, given that VFSS is widely considered to be the "gold standard" for assessment of swallow function. Certainly, direct visualization allows for an understanding of bolus flow and airway invasion that is not possible otherwise. Yet, the results of this study would appear to suggest that physical touch was a more sensitive modality than visual inspection for assessing HE. However, it is not possible to make any conclusive statements regarding the comparison of these two modalities due to limitations related to our retrospective design, the difference in raters across the two assessments, and lack of operationalization of the terms and parameters assessed.

One explanation for our findings may lie in the challenge of perceptually teasing apart different components of swallowing physiology. Judgment of HE may be largely influenced by clinicians' overall impression of swallow function, such that reduced HE may be "perceived" in a patient who demonstrates overt signs of airway invasion compared to one with no clinical signs or symptoms, despite minimal difference in objective HE between the two. Because reduced anterior HE has been shown to be significantly associated with higher risk of penetration-aspiration [29], the pairing of clinical ratings of "reduced" HE with reductions specifically in anterior movement might have occurred more frequently. However, this does not account for the lack of significant findings in VFSS ratings, which provide much more robust information about airway invasion.

Our suggestion that increased submental adipose tissue interferes with tactile perception of HE should also be considered in light of our results. We did not have information regarding neck circumference or other specific anthropometric measurements. However, the mean BMI of both males and females in this study, was over 25, classified by the World Health Organization as overweight [30]. It is possible that the higher BMI of our sample indicated larger body habitus and, correspondingly, increased submental tissue that affected palpation ability. Additional research is needed to explore the correlation between anthropometric measures and saliency of structural movement during swallowing.

The exploratory experience analysis was performed to determine whether clinician experience played a role in determining whether perceptual judgment of HE adequacy aligns with quantitative measures of hyoid excursion. We found no significant interaction between perceptual differentiation of objective measures and level of experience, suggesting that experience level was not a factor in clinicians' palpation sensitivity to greater and lesser magnitude of anterior hyoid movement. It is possible, however, that the relatively small number of observations in each group lacked sufficient power to yield results. Interestingly, clinicians with the least amount of experience (i.e. clinical fellows) were almost three times 
more likely to interpret $\mathrm{HE}$ as reduced when compared to the most experienced group (those with 10 or more years clinical experience). While this finding does not imply differences in palpation sensitivity between these two groups, it may indicate a tendency of novice clinicians towards more conservative judgment, potentially in instances where adequacy of $\mathrm{HE}$ is ambiguous.

Measures of HE were compared to values reported by Molfenter \& Steele [31]. Mean maximum XY position values for men and women across three volumes of thin liquid (5ml, $10 \mathrm{ml}$, and $20 \mathrm{ml})$ ranged from $147.6 \% \mathrm{C} 2-\mathrm{C} 4(95 \% \mathrm{CI}=140.3-154.9)$ for $5 \mathrm{ml}$ volumes in women to $157.9 \% \mathrm{C} 2-\mathrm{C} 4(95 \% \mathrm{CI}=150.6-165.2)$ for $20 \mathrm{ml}$ volumes in men. This range was markedly lower than the mean peak hypotenuse position in males $(186.8 \% \mathrm{C} 2-\mathrm{C} 4, \mathrm{SD}=$ $25.3)$ or overall $(187.1 \% \mathrm{C} 2-\mathrm{C} 4, \mathrm{SD}=25.4)$ in the current study. We believe this discrepancy is largely due to differences in participant age between the current study (mean age $=61.42$, $\mathrm{SD}=19.03$ ) and Molfenter and Steele (mean age $=31.5, \mathrm{SD}=5.7$ ). Changes in spinal morphology with age may affect the $\mathrm{C} 2-\mathrm{C} 4$ distance used to express hyoid movement. Cervical disc degeneration is present in $95 \%$ of people 65 years and older, and is associated with a loss of intervertebral disc height[32,33]. The vertebral body itself has also been shown to lose height with age, most notably at the level of C3-C6[34]. Therefore, absolute HE magnitude may not be necessarily larger in our sample, but is being expressed relative to a shorter $\mathrm{C} 2-\mathrm{C} 4$ length. Further research is needed to validate the use of the $\mathrm{C} 2-\mathrm{C} 4$ anatomical scalar in older populations.

For objective measurement of HE, the position of the hyoid bone was used to represent hyolaryngeal movement overall. Palmer et al. found strong, positive correlations between movements of the hyoid and larynx in both the horizontal $(r=.69)$ and vertical $(r=.63)$ planes of movement $[35,23]$, and Steele et al. found positive correlations $(r \geq 05)$ between hyoid bone and arytenoid movement for the majority of patients [29]. Therefore, measurement of hyoid bone movement was believed to be an adequate proxy for movement of the entire hyolaryngeal complex.

As previously noted, some patients had the same clinician perform the CSE and VFSS, and some were seen by a different clinician for each assessment. In either case, clinicians performing the VFSS were not blinded to the results of the CSE. Despite the lack of blinding and the majority $(54 / 87=62 \%)$ being rated by the same clinician within a 24 hour period, agreement between the two assessments was only moderate. In the acute care setting, the possibility of rapid changes in swallow function cannot be excluded. It is also likely that the inherent differences between visual and tactile information used to make judgments about HE contributed to the rating disagreement observed.

Additional limitations to those mentioned above are inherent to the reduced control over variables in this retrospective design. Quantitative peak hyoid measurements that were used for comparison to clinician ratings were collected on a single puree bolus per participant. While this was deemed necessary to control for bolus volume and viscosity, additional stimuli of varying viscosity and volume may have yielded more robust results, and would have been more representative of trials administered during the CSE. Another limitation was that judgment of normal on the CSE had to be indirectly assumed. When "reduced 
hyolaryngeal rise" was left unchecked on the CSE flowsheet with no additional comments on HE in the report, it was assumed that the clinician did not suspect impaired HE. There was also an unequal distribution of raters in each experience category for the exploratory analysis, and an unequal distribution of the number of assessments performed within each category. Lastly, we lacked rater training or a standard definition for what constituted "reduced" versus "functional" HE. Ratings were made via clinical decision-making based on past experience and training. This was believed to increase the ecological validity of the study, as nonstandard perceptual assessment and rating methods are common in clinical practice [19]. However, alternative scoring methods that use more standardized criteria for rating $\mathrm{HE}$ are becoming increasingly adopted. As mentioned, the MASA employs a fourpoint scale for HE adequacy during the CSE [20], and the MBSImP components 8 and 9 assess laryngeal and hyoid movement, respectively, during VFSS [36]. The use of standard definitions of HE movement and training clinician raters may have yielded different results. Given that all VFSS studies were recorded and available, it would have been possible to have HE re-assessed by a trained rater using standardized methods. The comparison of these ratings to those made in real time may prove to be an interesting additional analysis in the future.

Overall, the findings of this investigation demonstrated no clear differentiation of quantitative measures of HE based on perceptual evaluation. Clinicians may choose to use palpation to gain information such as presence or absence of a swallow and the number of swallows per bolus, and certainly, visual review of VFSS should consider the relative movement of all structures during the swallow. Nevertheless, although additional, prospective research is needed, this study suggests that awareness of the potential limitations of subjective judgment for detecting HE pathology should be reflected in how clinical findings are interpreted and documented, and in the extent to which this specific component of the swallow evaluation influences clinical impressions, recommendations, and treatment plans. While keeping in mind that hyolaryngeal movement is only one piece of the diagnostic puzzle, it is possible that factors beyond peak excursion, such as velocity and duration of movement, may factor into clinical perception of pathology. These factors and others should be explored in future research.

\section{Conclusion}

We conducted this study to better understand the role of perceptual methods in clinically evaluating HE. Overall, clinician perception of reduced and normal HE was minimally associated with actual hyoid movement, however, findings from this study indicated increased sensitivity of palpation over VFSS visualization for assessing adequacy of HE. There was no effect of clinical experience on palpation sensitivity. Perceptual assessment techniques will likely continue to factor heavily in the evaluation of swallowing, particularly in settings where other assessment tools are not available or feasible. However, the results of this study suggest that clinicians should remain guarded when making clinical judgments about adequacy of hyolaryngeal movement. 


\section{Acknowledgements:}

The authors would like to thank James C. Borders for his assistance during data collection, Glen Leverson and Sharon Weinberg for their assistance during data analysis, and the speech language pathologists at UW-Madison Voice and Swallow clinics. Portions of this work were presented at the 2018 Dysphagia Research Society meeting. The authors declare no conflict of interest to disclose.

Funding: Diane M. Bless Endowed Chair in Otolaryngology, University of Wisonsin- Madison

\section{References}

1. Dodds WJ, Stewart ET, Logemann JA (1990) Physiology and radiology of the normal oral and pharyngeal phases of swallowing. AJR Am J Roentgenol 154 (5):953-963. doi:10.2214/ajr. 154.5.2108569 [PubMed: 2108569]

2. Matsuo K, Palmer JB (2008) Anatomy and physiology of feeding and swallowing: normal and abnormal. Phys Med Rehabil Clin N Am 19 (4):691-707, vii. doi:10.1016/j.pmr.2008.06.001 [PubMed: 18940636]

3. Paik NJ, Kim SJ, Lee HJ, Jeon JY, Lim JY, Han TR (2008) Movement of the hyoid bone and the epiglottis during swallowing in patients with dysphagia from different etiologies. J Electromyogr Kines 18 (2):329-335. doi:10.1016/j.jelekin.2006.09.011

4. Logemann JA, Kahrilas PJ, Cheng J, Pauloski BR, Gibbons PJ, Rademaker AW, Lin S (1992) Closure mechanisms of laryngeal vestibule during swallow. Am J Physiol 262 (2 Pt 1):G338-344. doi:10.1152/ajpgi.1992.262.2.G338 [PubMed: 1539666]

5. Jacob P, Kahrilas PJ, Logemann JA, Shah V, Ha T (1989) Upper esophageal sphincter opening and modulation during swallowing. Gastroenterology 97 (6):1469-1478 [PubMed: 2583413]

6. McCullough GH, Rosenbek JC, Wertz RT, McCoy S, Mann G, McCullough K (2005) Utility of clinical swallowing examination measures for detecting aspiration post-stroke. J Speech Lang Hear Res 48 (6):1280-1293. doi:10.1044/1092-4388(2005/089) [PubMed: 16478371]

7. Ramsey DJC, Smithard DG, Kalra L (2003) Early assessments of dysphagia and aspiration risk in acute stroke patients. Stroke 34 (5):1252-1257. doi:10.1161/01.Str.0000066309.06490.B8 [PubMed: 12677020]

8. Logemann JA (1998) Evaluation and Treatment of Swallowing Disorders, 2nd edition. Pro-Ed, Austin, TX

9. McCullough GH, \& Martino R (2013) Clinical evaluation of patients with dysphagia: importance of history taking and physical exam In: Shaker E, C. R, Belafsky PC, \& Postma GN (ed) Manual of Diagnostic and Therapeutic Techniques for Disorders of Deglutition. Springer-Verlag, New York, pp 11-30

10. Ishida R, Palmer JB, Hiiemae KM (2002) Hyoid motion during swallowing: factors affecting forward and upward displacement. Dysphagia 17 (4):262-272. doi:10.1007/s00455-002-0064-5 [PubMed: 12355141]

11. Kim Y, McCullough GH (2008) Maximum hyoid displacement in normal swallowing. Dysphagia 23 (3):274-279. doi:10.1007/s00455-007-9135-y [PubMed: 17962998]

12. Chi-Fishman G, Sonies BC (2002) Effects of systematic bolus viscosity and volume changes on hyoid movement kinematics. Dysphagia 17 (4):278-287. doi:10.1007/s00455-002-0070-7 [PubMed: 12355143]

13. Nagy A, Molfenter SM, Peladeau-Pigeon M, Stokely S, Steele CM (2014) The effect of bolus volume on hyoid kinematics in healthy swallowing. Biomed Res Int 2014:738971. doi: 10.1155/2014/738971 [PubMed: 24779015]

14. Rofes L, Arreola V, Martin A, Clave P (2013) Natural capsaicinoids improve swallow response in older patients with oropharyngeal dysphagia. Gut 62 (9):1280-1287. doi:10.1136/ gutjnl-2011-300753 [PubMed: 22722616]

15. Leow LP, Huckabee ML, Sharma S, Tooley TP (2007) The influence of taste on swallowing apnea, oral preparation time, and duration and amplitude of submental muscle contraction. Chem Senses 32 (2):119-128. doi:10.1093/chemse/bj1037 [PubMed: 17071940] 
16. Leonard RJ, Kendall KA, McKenzie S, Goncalves MI, Walker A (2000) Structural displacements in normal swallowing: a videofluoroscopic study. Dysphagia 15 (3):146-152. doi:10.1007/ s004550010017 [PubMed: 10839828]

17. Logemann JA, Pauloski BR, Rademaker AW, Colangelo LA, Kahrilas PJ, Smith CH (2000) Temporal and biomechanical characteristics of oropharyngeal swallow in younger and older men. $\mathrm{J}$ Speech Lang Hear Res 43 (5):1264-1274 [PubMed: 11063246]

18. Molfenter SM, Steele CM (2011) Physiological variability in the deglutition literature: hyoid and laryngeal kinematics. Dysphagia 26 (1):67-74. doi:10.1007/s00455-010-9309-x [PubMed: 20927634]

19. McCullough GH, Wertz RT, Rosenbek JC, Dinneen C (1999) Clinicians' preferences and practices in conducting clinical-bedside and videofluoroscopic swallowing examinations in an adult, neurogenic population. American Journal of Speech-Language Pathology 8 (2):149-163

20. Mann G (2002) MASA: The Mann Assessment of Swallowing Ability, vol 1 Cengage Learning,

21. McCullough GH, Wertz RT, Rosenbek JC, Mills RH, Ross KB, Ashford JR (2000) Inter- and intrajudge reliability of a clinical examination of swallowing in adults. Dysphagia 15 (2):58-67. doi:10.1007/s004550010002 [PubMed: 10758187]

22. Rangarathnam B, McCullough GH (2016) Utility of a Clinical Swallowing Exam for Understanding Swallowing Physiology. Dysphagia 31 (4):491-497. doi:10.1007/ s00455-016-9702-1 Perceptual methods for rating hyolaryngeal excursion [PubMed: 26970759]

23. Lee JW, Randall DR, Evangelista LM, Kuhn MA, Belafsky PC (2017) Subjective Assessment of Videofluoroscopic Swallow Studies. Otolaryngol Head Neck Surg 156 (5):901-905. doi: 10.1177/0194599817691276 [PubMed: 28195753]

24. Schneider CA, Rasband WS, Eliceiri KW (2012) NIH Image to ImageJ: 25 years of image analysis. Nat Methods 9 (7):671-675 [PubMed: 22930834]

25. Molfenter SM, Steele CM (2014) Use of an Anatomical Scalar to Control for Sex- Based Size Differences in Measures of Hyoid Excursion During Swallowing. J Speech Lang Hear R 57 (3): 768-778. doi:10.1044/2014_Jslhr-S-13-0152

26. Perlman AL, VanDaele DJ, Otterbacher MS (1995) Quantitative assessment of hyoid bone displacement from video images during swallowing. J Speech Hear Res 38 (3):579-585 [PubMed: 7674650]

27. Thompson TZ, Obeidin F, Davidoff AA, Hightower CL, Johnson CZ, Rice SL, Sokolove RL, Taylor BK, Tuck JM, Pearson WG Jr., (2014) Coordinate mapping of hyolaryngeal mechanics in swallowing. J Vis Exp (87). doi:10.3791/51476

28. Fleiss JL (1986) The design and analysis of clinical experiments. Wiley, New York, NY

29. Steele CM, Bailey GL, Chau T, Molfenter SM, Oshalla M, Waito AA, Zoratto DC (2011) The relationship between hyoid and laryngeal displacement and swallowing impairment. Clin Otolaryngol 36 (1):30-36. doi:10.1111/j.1749-4486.2010.02219.x [PubMed: 21414151]

30. Organization WH (1995) Physical Status: The Use and Interpretation of Anthropometry Technical Report Series, vol 854 Geneva, Switzerland

31. Molfenter SM, Steele CM (2014) Use of an Anatomical Scalar to Control for Sex- Based Size Differences in Measures of Hyoid Excursion During Swallowing. Journal of Speech, Language \& Hearing Research 57 (3):768-778. doi:10.1044/2014_JSLHR-S-13-0152

32. Benoist M (2003) Natural history of the aging spine. Eur Spine J 12 Suppl 2:S86-89. doi:10.1007/ s00586-003-0593-0 [PubMed: 12961079]

33. Malcolm GP (2002) Surgical disorders of the cervical spine: presentation and management of common disorders. J Neurol Neurosurg Psychiatry 73 Suppl 1:i34-41 [PubMed: 12185260]

34. Ezra D, Masharawi Y, Salame K, Slon V, Alperovitch-Najenson D, Hershkovitz I (2017) Demographic aspects in cervical vertebral bodies' size and shape (C3-C7): a skeletal study. Spine J 17 (1):135-142. doi:10.1016/j.spinee.2016.08.022 [PubMed: 27544049]

35. Palmer JB, Tanaka E, Ensrud E (2000) Motions of the posterior pharyngeal wall in human swallowing: a quantitative videofluorographic study. Arch Phys Med Rehabil 81 (11):1520-1526. doi:10.1053/apmr.2000.17829 [PubMed: 11083359] 
36. Martin-Harris B, Brodsky MB, Michel Y, Castell DO, Schleicher M, Sandidge J, Maxwell R, Blair J (2008) MBS measurement tool for swallow impairment--MBSImp: establishing a standard. Dysphagia 23 (4):392-405. doi:10.1007/s00455-008-9185-9 [PubMed: 18855050] 


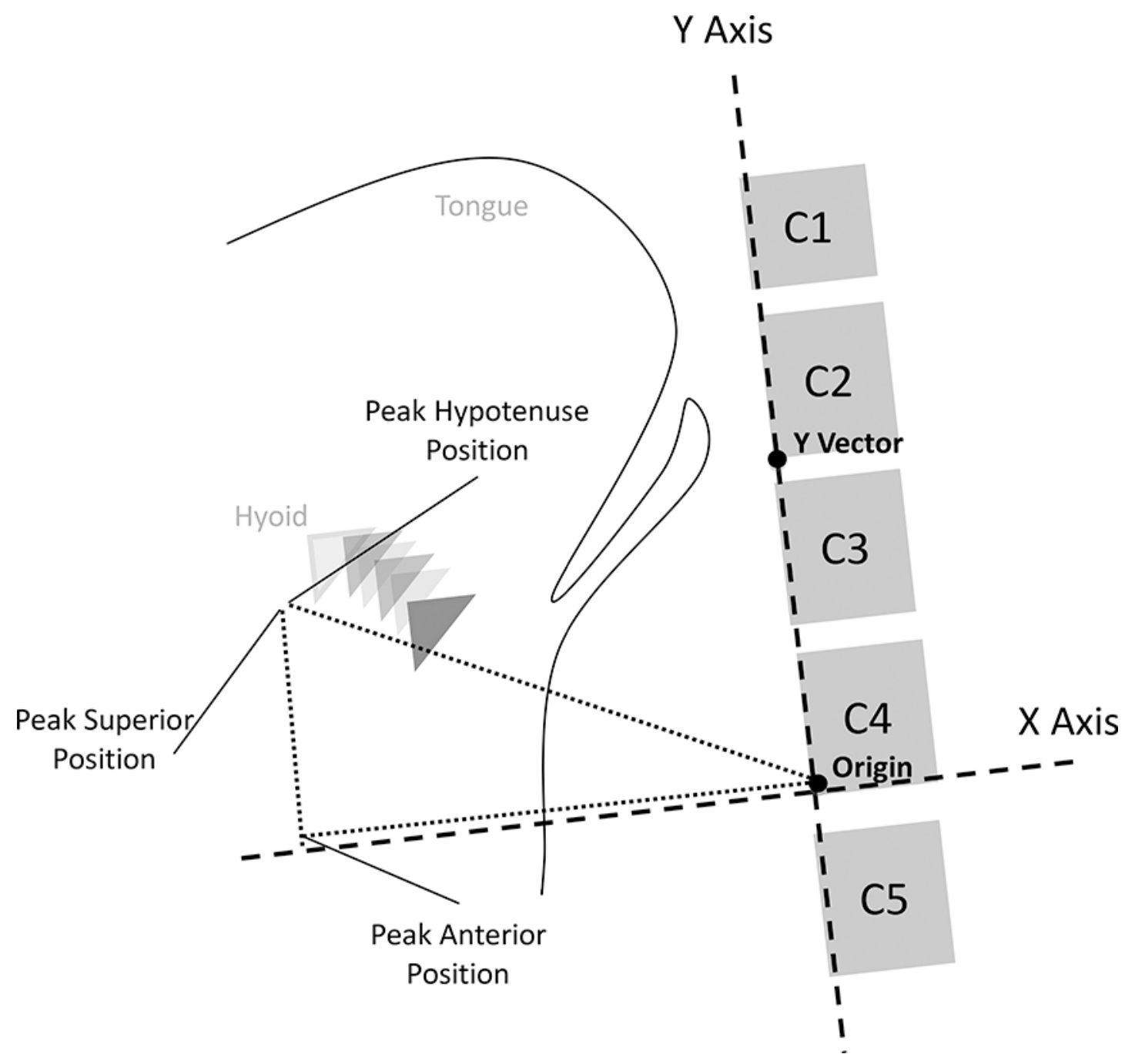

Fig. 1.

Representation of the measurement parameters for measuring hyoid distance from $\mathrm{C} 4$ at the height of the swallow. Source: Molfenter \& Steele [24]. 


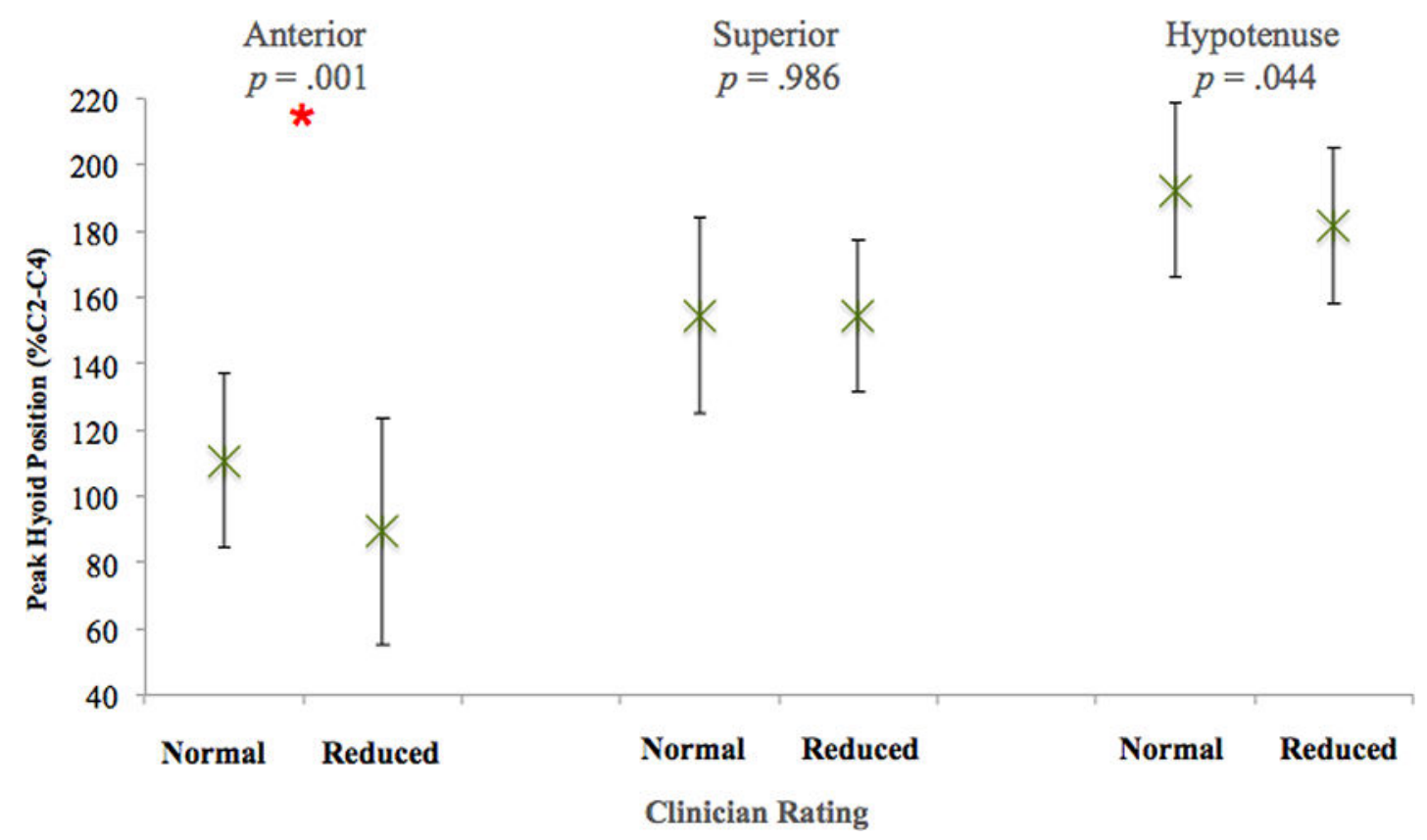

Fig. 2.

Comparison of CSE ratings to peak hyoid measures. Asterisks represent mean values, vertical lines represent standard deviations; ${ }^{*}=\mathrm{p}<0.008$ 


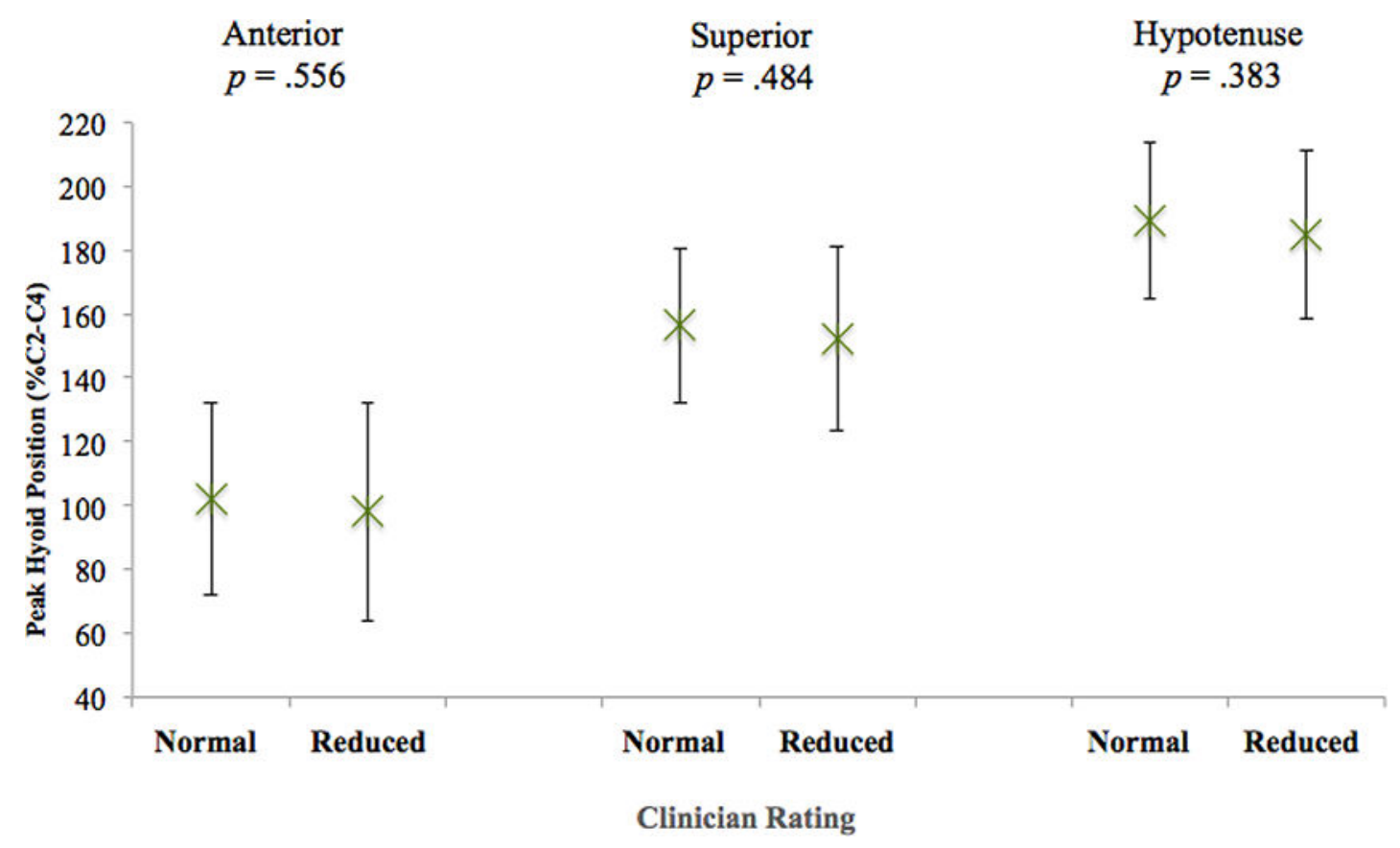

Fig. 3.

Comparison of VFSS ratings to peak hyoid measures. Asterisks represent mean values, vertical lines represent standard deviations; $\mathrm{p}<0.008$ 
Table 1

Clinician Experience Groups

\begin{tabular}{|rrrr|}
\hline Group & Years Experience & No. Raters & No. Combined CSEs performed \\
\hline 1 & 1 year or less & 7 & 33 \\
2 & $1-10 \mathrm{yrs}$ & 5 & 18 \\
3 & $10+$ years & 4 & 36 \\
\hline
\end{tabular}




\section{Table 2}

\begin{tabular}{|c|c|c|}
\hline \multirow{9}{*}{ 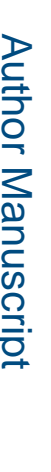 } & \multicolumn{2}{|c|}{ Inpatient Service Category } \\
\hline & Service & $\mathbf{N}(\%)$ \\
\hline & Neurology/Neurosurgery & $26(29.8)$ \\
\hline & Hospitalist/General Medicine & $24(27.6)$ \\
\hline & Trauma & $12(13.8)$ \\
\hline & Other ( $\mathcal{Q}$ per category) ${ }^{*}$ & $11(12.6)$ \\
\hline & Cardiovascular & $8(9.2)$ \\
\hline & Oncology & $3(3.4)$ \\
\hline & Transplant & $3(3.4)$ \\
\hline
\end{tabular}

* Other= critical care, orthopedic surgery, otolaryngology, hematology, urology, palliative care, advanced pulmonary service, general surgery 


\section{Table 3}

Reliability across hyoid measures

\begin{tabular}{|c|c|c|c|}
\hline & Anterior & Superior & Hypotenuse \\
\hline $\begin{array}{c}\text { Intra-rater } \\
\text { (ICC) }\end{array}$ & .996 & .989 & .990 \\
\hline $\begin{array}{c}\text { Inter-rater } \\
\text { (ICC) }\end{array}$ & .923 & .950 & .892 \\
\hline
\end{tabular}

ICC $=$ Intra-class correlation coefficients 


\section{Table 4}

Distribution of ratings across perceptual categories

\begin{tabular}{|c|c|c|c|}
\hline & N-VF & R-VF & Total \\
\hline N-CSE & 29 & 16 & 45 \\
\hline R-CSE & 17 & 25 & 42 \\
\hline Total & 46 & 41 & 87 \\
\hline
\end{tabular}

$\mathrm{N}-\mathrm{CSE}=$ rated as within normal limits during $\mathrm{CSE} ; \mathrm{R}-\mathrm{CSE}=$ rated as reduced at $\mathrm{CSE} ; \mathrm{N}-\mathrm{VF}=$ rated as within normal limits during VF; $\mathrm{R}-\mathrm{VF}=$ rated as reduced at $\mathrm{VF}$ 


\section{Table 5}

Descriptive statistics of objective measures of HE within each group

\begin{tabular}{|l|c|c|c|c|c|}
\hline \multicolumn{7}{|l|}{ Hyoid Measures } \\
\hline (\%C2-C4 distance)N-CSE & N-CSE & R-CSE & N-VF & R-VF & All \\
\hline Peak Anterior M(SD) & $110.6(26.18)^{*}$ & $89.22(34.1)^{*}$ & $102.2(30.1)$ & $98.1(34.2)$ & $100.3(31.9)$ \\
\hline Peak Superior M(SD) & $154.5(29.5)$ & $154.4(23)$ & $156.4(24.3)$ & $152.3(28.8)$ & $154.5(26.4)$ \\
\hline Peak Hypotenuse M(SD) & $192.4(26.3)$ & $181.4(23.4)$ & $189.1(24.4)$ & $184.8(26.5)$ & $187.1(25.4)$ \\
\hline
\end{tabular}

$\mathrm{N}-\mathrm{CSE}=$ rated as within normal limits during $\mathrm{CSE}$; $\mathrm{R}-\mathrm{CSE}=$ rated as reduced at $\mathrm{CSE} ; \mathrm{N}-\mathrm{VF}=$ rated as within normal limits during $\mathrm{VF} ; \mathrm{R}-\mathrm{VF}=$ rated as reduced at $\mathrm{VF}$

* $=\mathrm{p}<.008$ 
Table 6

Perceptual Agreement Between CSE and VF by Consistency of Rater

\begin{tabular}{lrrr}
\hline & Same Rater (n) & Different Rater (n) & Total (n) \\
\hline Agreement & 42 & 23 & 65 \\
No Agreement & 12 & 10 & 22 \\
Total & 54 & 33 & 87 \\
\hline
\end{tabular}

